\title{
Radiosurgery target location and individual anatomical variation in trigeminal nerves
}

\author{
Clinical article
}

\author{
Yi-Chieh Hung, M.D., ${ }^{1,3}$ Cheng-Chia Lee, M.D., ${ }^{2,3}$ KAng-Du Liu, M.D., $, 2,3$ \\ Wen-Yuh Chung, M.D., ${ }^{2,3}$ David Hung-Chi Pan, M.D., ${ }^{2-4}$ And Huai-Che Yang, M.D. ${ }^{2,3}$ \\ ${ }^{1}$ Division of Neurosurgery, Department of Surgery, Chi-Mei Foundation Hospital, Tainan; ${ }^{2}$ Department of \\ Neurosurgery, Neurological Institute, Taipei Veterans General Hospital; ${ }^{3}$ School of Medicine, National \\ Yang-Ming University, Taipei; and ${ }^{4}$ Department of Neurosurgery, Taipei Medical University-Shuang Ho \\ Hospital, New Taipei, Taiwan
}

\begin{abstract}
Object. The authors evaluated individual anatomical variations in the trigeminal nerves of patients with medically intractable trigeminal neuralgia and clarified the relationships among the variations, radiosurgical target locations, and the clinical outcomes after high-dose Gamma Knife surgery (GKS).

Methods. From 2006 through 2011, the authors conducted a retrospective review of 106 cases of primary or secondary trigeminal neuralgia consecutively treated with GKS targeting the dorsal root entry zone (DREZ) for which a maximal dose of $90 \mathrm{~Gy}$ and a $20 \%$ isodose line to the brainstem were used. A questionnaire was used to evaluate patients' pre- and post-GKS clinical conditions. To evaluate individual anatomical variations among trigeminal nerves, the authors used 3 parameters: the length of the trigeminal nerve in the cistern (nerve length), the length of the target between the radiation shot and the brainstem (targeting length), and the ratio between nerve length and targeting length (targeting ratio).

Results. The median length of the trigeminal nerves in the 106 patients was $9.6 \mathrm{~mm}$ (range $6.04-20.74 \mathrm{~mm}$ ), the median targeting length was $3.8 \mathrm{~mm}$ (range 1.81-10.84 $\mathrm{mm}$ ), and the median targeting ratio was 38\% (range $13 \%$ $80 \%$ ). No statistically significant differences in pain relief and pain recurrence were detected among patients with these various nerve characteristics. However, radiation-induced facial hypesthesia correlated with nerve length and targeting ratio $(\mathrm{p}<0.05)$ but not with absolute distance from the brainstem (targeting length).

Conclusions. In trigeminal neuralgia patients who received DREZ-targeted GKS, the rate of pain relief did not differ according to anatomical nerve variations. However, the frequency of facial hypesthesia was higher among patients in whom the nerve was longer $(>11 \mathrm{~mm})$ or the targeting ratio was lower $(<36 \%)$. Adjusting the target according to the targeting ratio, especially for patients with longer nerves, can reduce facial hypesthesia and enable maintenance of effective pain control.
\end{abstract}

(http://thejns.org/doi/abs/10.3171/2014.7.GKS141432)

\section{Key Words • Gamma Knife • trigeminal neuralgia • radiosurgery • radiation-induced facial numbness - stereotactic radiosurgery}

$\mathrm{L}$ ARS Leksell first used stereotactic radiosurgery to treat trigeminal neuralgia in 1951,9 and each year during the past decade, more than 3000 patients with trigeminal neuralgia have undergone treatment with this new modality. ${ }^{19}$ The efficacy and limitations of Gamma Knife surgery (GKS) for patients with trigeminal neuralgia have been addressed in several articles. Higher radiation dosages were used in only a few studies and correlated with more successful pain control and lower rates of pain recurrence but with high percentages of facial hypesthesia and nerve damage. , $, 7,10,24^{2}$

Abbreviations used in this paper: BNI = Barrow Neurological Institute; DREZ = dorsal root entry zone; GKS = Gamma Knife surgery; RGZ = retrogasserian zone.
Although identifying the ideal targeting location is crucial, no consensus on an ideal radiosurgical target for patients with trigeminal neuralgia has been achieved. Most radiosurgeons have administered radiation to the dorsal root entry zone (DREZ), ${ }^{2,4,12,22}$ which is generally considered to be the area $3 \mathrm{~mm}$ anterior to the location at which the nerve emerges from the pons. ${ }^{1}$ According to studies in which the DREZ ${ }^{2,4,12,22}$ and the region 2-4 $\mathrm{mm}$ from the brainstem ${ }^{21}$ were targeted, the initial rates of freedom from pain ranged from $70 \%$ to $96.3 \%$, and the incidence of the development of facial hypesthesia ranged from $6 \%$ to $37 \%$. Several researchers have proposed that the retrogasserian zone (RGZ) rather than the DREZ can be targeted to increase pain relief and reduce facial hypesthesia. The initial rates of freedom from pain for pa- 
Y. C. Hung et al.

tients receiving RGZ-targeted GKS ranged from $71 \%$ to $97 \%$, and the incidence of trigeminal nerve injury was $10 \%$ to $38 \% .^{10,17,18}$ Because of discrepancies among the methods used in different studies, determining the ideal target according to current evidence is difficult.

Another essential factor observed in our daily practice is variation in the length of trigeminal nerves. At Taipei Veteran General Hospital, the measured lengths of trigeminal nerves in the cerebellopontine cistern range from 6.0 to $20.7 \mathrm{~mm}$. Because of these variations in nerve length, performing GKS by using a constant distance from the brainstem can result in irradiation of different segments of the trigeminal nerve. To the best of our knowledge, previous studies have not adequately evaluated the role of anatomical variations.

Our objectives with this study were to evaluate results of high-dose GKS used to treat medically intractable trigeminal neuralgia, clarify the role of individual anatomical variations with regard to treatment, and propose a reasonable method for adjusting GKS targeting.

\section{Methods}

\section{Patient Population}

From 1993 through 2011, a series of 307 patients with idiopathic or secondary trigeminal neuralgia consecutively underwent GKS at our center (Taipei Veterans General Hospital). The indications for radiosurgery were salient pain despite prior aggressive medical therapy or intolerance of the adverse effects of prescribed medication. From 2006 through 2011, among 134 patients who had been treated during this period and reviewed for inclusion in our study, a total of 106 patients met the study inclusion criteria. Among these 106 patients, 36 were men and 70 were women; mean age was 64 years (range 3388 years); median follow-up period after GKS was 41.5 months (range 12-83 months); and 33 had undergone at least 1 previous surgical procedure (of which 10 procedures were GKS performed at other institutions), and the other 73 were unwilling or unable to undergo surgical interventions. Excluded from the study were patients who had died, were lost to follow-up, or were unable to describe their symptoms (Table 1).

\section{GKS}

All patients underwent radiosurgery performed with the Leksell Gamma Unit model C (Elekta AB) as well as stereotactic MRI conducted by using a 1.5-T Siemens imaging unit after a Leksell stereotactic frame had been fixed to the patient's head. For delineation of the trigeminal nerve and its relationship to the surrounding vasculature, thin-section (1.5-mm), noncontrast T1-weighted and T2-weighted images were obtained. All treatment plans used a single 4-mm isocenter with no blocks. Generally, the isocenter was placed $4 \mathrm{~mm}$ anterior to the brainstem and adjusted according to the $20 \%$ isodose line tangential to the pontine surface. The maximal dose administered to the brainstem surface was $18 \mathrm{~Gy}$. The mean maximal dose administered to the trigeminal nerve was $88 \mathrm{~Gy}$ (median 90 Gy, range 70-90 Gy) (Table 1).
TABLE 1: Patient and clinical characteristics*

\begin{tabular}{lc}
\hline \multicolumn{1}{c}{ Characteristic } & Value (range) \\
\hline male/female ratio & $36: 70$ \\
mean/median age in yrs & $64 / 64(33-88)$ \\
side of pain, rt/lt & $68 / 38$ \\
distribution of pain & \\
V1 only/V2 only/V3 only & $1 / 25 / 23$ \\
V2 + V3 & 43 \\
V1 + V2 & 5 \\
V1 + V2 + V3 & 9 \\
previous operation, no/yes & $73 / 33$ \\
MVD & 19 \\
GKS & 10 \\
rhizotomy & 5 \\
$\geq 2$ operations & 9 \\
mean/median maximal dose in Gy & $88 / 90(70-90)$ \\
mean/median follow-up in mos & $44.6 / 41.5(12-83)$ \\
\hline
\end{tabular}

* MVD = microvascular decompression; V1 = first branch of trigeminal nerve; V2 = second branch of trigeminal nerve; V3 = third branch of trigeminal nerve.

\section{Patient Follow-Up and Outcome Evaluations}

After GKS, all patients underwent at least 1 year of follow-up evaluation in 3- to 6-month intervals. A questionnaire designed to evaluate patients' pre- and postGKS clinical conditions was administered by telephone. The questionnaire addressed the degree of pain relief, the patterns of facial pain before treatment, the time interval between treatment and pain relief, the patient's use of medication, and the degree of facial hypesthesia. To avoid selection bias between the surgeons and patients, the telephone questionnaires were administered by 3 technicians from our center's Gamma Knife Team who were not involved in patient selection or management.

The patients' pain outcomes were scored according to the Barrow Neurological Institute (BNI) pain intensity scale. ${ }^{20}$ We defined BNI pain intensity Score I as complete pain relief, Scores I-IIIb as adequate pain relief, and Scores IV and V as treatment failures. Because facial hypesthesia has been frequently observed after high-dose radiosurgery, according to previous studies, ${ }^{8,16,17}$ we used the BNI facial hypesthesia scale to evaluate patients' radiation-induced facial hypesthesia. For statistical analyses, facial hypesthesia with BNI scores of II or III was defined as positive facial hypesthesia.

\section{Evaluation of Nerve Characteristics}

To evaluate whether differences in nerve characteristics and target locations cause variations in clinical outcomes, we defined 3 parameters: the length of the trigeminal nerve in the cistern (nerve length), the length of the target between the radiation shot and brainstem (targeting length), and the ratio between the nerve length and the targeting length (targeting ratio). We used Leksell GammaPlan software (Elekta AB) to obtain the coordinates of the midpoint of the junction between the trigeminal 


\section{Radiosurgery targeting and anatomical trigeminal nerve variation}

nerve and the brainstem, radiation shot of the Gamma Knife, and the midpoint of trigeminal nerve as it reaches the dural edge (Fig. 1). The nerve length was calculated as the distance from the brainstem to the dural edge. The targeting length was calculated as the distance from the brainstem to the shot of the Gamma Knife. The targeting ratio was calculated by using simple division: targeting length/nerve length $=$ targeting ratio.

\section{Statistical Analysis}

Data are presented as the median (range) or mean $( \pm \mathrm{SD})$ for continuous variables and as frequencies and percentages for categorical variables. Cox regression analyses and hazard ratios were used to evaluate the time to response and the duration of pain relief in relation to the 3 characteristics (nerve length, targeting length, and targeting ratio). Logistic regression and odds ratios were used to evaluate the relationship between facial hypesthesia and the 3 nerve characteristics. Probability values less than 0.05 indicated statistical significance. For statistical analyses, we used SPSS, version 17.0 (SPSS Inc.).

\section{Results}

\section{Effect of GKS on Pain Relief}

After a median latency period of 2 months (range 1-18 months) after GKS, 87 (82\%) patients exhibited complete or adequate pain relief (BNI pain intensity Scores I-IIIb), $19(18 \%)$ reported poor pain relief (BNI Score IV or V), and $59(56 \%)$ exhibited complete pain relief (BNI Score
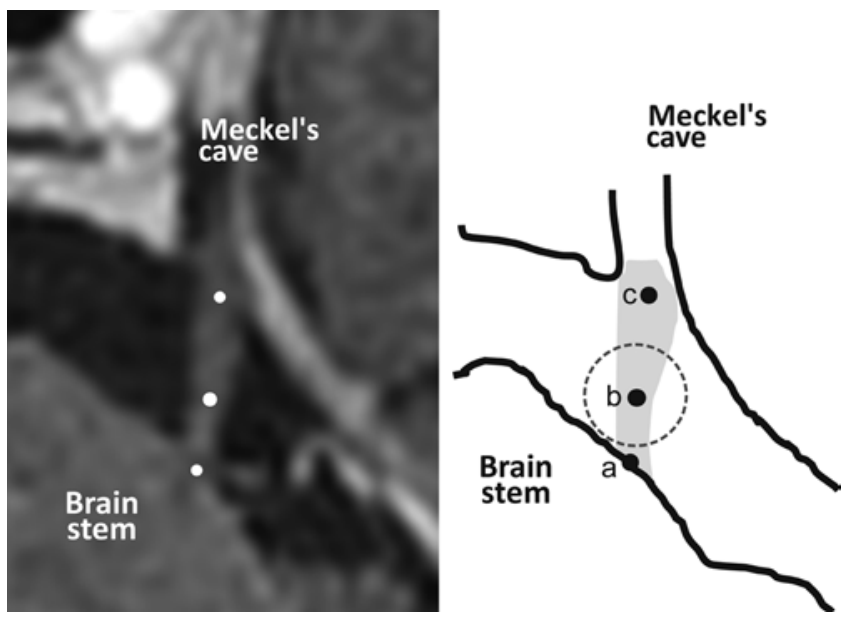

FIG. 1. Left: T1-weighted MR image with labels and marks showing a left trigeminal nerve and its relationship to the surrounding structures. The 3 white dots represent the coordinates ( $a, b$, and $c)$ shown on the right. Right: Illustration and definitions of nerve length, targeting length, and targeting ratio. The coordinates of the midpoint of the junction between the trigeminal nerve and the brainstem (a), radiation shot of the Gamma Knife (b) with a $20 \%$ isodose line to brainstem (dashed circle), and midpoint of the trigeminal nerve as it reaches the dural edge (c) were obtained by using the Leksell GammaPlan software. Nerve length was calculated as the distance between the brainstem and the dural edge (nerve length $=a-c$ ). Targeting length was calculated as the distance from the brainstem to the shot of the Gamma Knife (targeting length $=a-b)$. Targeting ratio was calculated by dividing the targeting length by the nerve length (targeting ratio $=a-b / a-c$ ).
I). We evaluated the duration of pain relief after the initial response by using the Kaplan-Meier product-limit method. Among the 87 patients who had complete or adequate pain relief, 15 patients experienced pain recurrence at a median of 12 months (range 3-24 months) (Table 2).

\section{Facial Hypesthesia and Other Complications After GKS}

No acute post-GKS neurological complications were noted. Table 3 shows the BNI scores for facial hypesthesia. Among the 106 treated patients, 57 (54\%) reported new facial hypesthesia after GKS: BNI facial numbness Score II for 44 (42\%), BNI Score III for 13 (12\%), and BNI Score IV for none. Among the 59 patients who reported complete pain relief (BNI Score I), 22 (37\%) reported no facial hypesthesia, $27(46 \%)$ received a BNI facial numbness score of II, and $10(17 \%)$ received a BNI score of III. Among the 87 patients who reported adequate pain relief (BNI Score I-IIIb), 39 (45\%) patients reported no facial hypesthesia, 37 $(43 \%)$ received a BNI facial numbness score of II, and 11 $(12 \%)$ received a BNI score of III (Table 3).

No other complications (e.g., mastication muscle weakness, hearing impairment, decreased corneal sensation, facial weakness, or anesthesia dolorosa) were reported.

\section{Association Between Nerve Characteristics and Clinical Outcomes}

The median length of the trigeminal nerve was 9.6

TABLE 2: Outcomes for patients who underwent GKS for trigeminal neuralgia*

\begin{tabular}{lc}
\hline \multicolumn{1}{c}{ Treatment Outcomes } & Value \\
\hline initial pain response, no. (\%) & $59(56)$ \\
BNI Grade I & $1(1)$ \\
BNI Grade II & $9(8)$ \\
BNI Grade IIla & $18(17)$ \\
BNI Grade IIIb & $1(1)$ \\
BNI Grade IV & $18(17)$ \\
BNI Grade V & $19(18)$ \\
initial treatment failure, no. (\%) & $2(1-18)$ \\
median time to initial response (range), mos & $15(14)$ \\
recurrence of pain after initial pain relief, no. (\%) & $12(3-24)$ \\
median time of pain recurrence (range), mos & $57(54)$ \\
clinically remarkable facial sensory dysfunction, & \\
no. (\%) & \\
characteristics of trigeminal NL, TL, \& TR & \\
NL in mm & \\
median (range) & \\
$\quad$ mean \pm SD & $10.1 \pm 3.9$ \\
TL in mm & \\
median (range) & $38(13-80)$ \\
mean \pm SD & $40 \pm 12$ \\
TR in \% & $3.9 \pm 1.31-10.84)$ \\
median (range) & \\
mean \pm SD & \\
\hline
\end{tabular}

\footnotetext{
${ }^{*} \mathrm{NL}=$ nerve length; $\mathrm{TL}=$ targeting length; $\mathrm{TR}=$ targeting ratio.
} 
TABLE 3: Correlation between post-GKS facial hypesthesia and facial pain intensity

\begin{tabular}{|c|c|c|c|c|c|}
\hline \multirow[b]{2}{*}{ BNI Score } & \multirow[b]{2}{*}{ Description } & \multicolumn{4}{|c|}{ No. $(\%)$} \\
\hline & & Total & $\mathrm{BNI} \mathrm{I}^{*}$ & BNI I-IIIb† & BNI IV-V $\ddagger$ \\
\hline I & no facial hypesthesia & $49(46)$ & 22 & 39 & 10 \\
\hline$\|$ & mild facial hypesthesia, not bothersome & $44(42)$ & 27 & 37 & 7 \\
\hline III & somewhat bothersome facial hypesthesia & $13(12)$ & 10 & 11 & 2 \\
\hline IV & very bothersome facial hypesthesia & 0 & 0 & 0 & 0 \\
\hline
\end{tabular}

$\mathrm{mm}$ (range 6.04-20.74 $\mathrm{mm}$ ), the median targeting length was $3.8 \mathrm{~mm}$ (range 1.81-10.84 $\mathrm{mm}$ ), and the median targeting ratio was $38 \%$ (range 13\%-80\%) (Table 2). Clinical factors, including complete pain relief, adequate pain relief, recurrence of symptoms, and facial hypesthesia, were assessed for the purpose of determining correlations with the 3 nerve characteristics. Facial hypesthesia was more commonly observed among patients with a longer nerve and a lower targeting ratio $(\mathrm{p}=0.008$ and 0.039 , respectively, Table 4). No statistical significance was observed between individual anatomical variations and the outcomes of pain relief and pain recurrence.

According to logistic regression analyses, the odds ratio between the nerve length and facial hypesthesia was 1.248 (with adjustment for age and sex, $\mathrm{p}<0.05$ ), and the odds ratio between targeting ratio and facial hypesthesia was 0.965 (with adjustment for age and sex, p < 0.05 , Table 4). After adjustment for age and sex, the risk for new facial hypesthesia increased by $24.5 \%$ among patients whose nerve length was $1 \mathrm{~mm}$ longer than the mean nerve length; furthermore, the risk for new facial hypesthesia increased by $3.4 \%$ for each $1.0 \%$ decrease in the targeting ratio (Table 4).

\section{Discussion}

Craniometry and Trigeminal Neuralgia

Individual variations in trigeminal nerves have been revealed in several studies. Régis et al. reported that among 100 patients, the median length of the trigeminal nerve in the cistern was $11.6 \mathrm{~mm}$ (range 3.3-21.2 mm). ${ }^{18}$ Kakizawa et al. measured 220 trigeminal nerves in 110 patients who had undergone brain MRI for reasons other than trigeminal neuralgia and found mean length of the trigeminal nerves to be $9.66 \pm 1.71 \mathrm{~mm}$ (range 5.53-14.1 $\mathrm{mm}) .{ }^{6}$ Park et al. compared the anatomical variations in 26 patients with trigeminal neuralgia and found the mean length of the cisternal segment of the trigeminal nerve to be $7.9 \mathrm{~mm}$ (range $4.9-14.2 \mathrm{~mm}$ ) on the affected side and $9.6 \mathrm{~mm}$ (range $6.7-12.8 \mathrm{~mm}$ ) on the nonaffected side. ${ }^{15}$ Parise et al. conducted an anatomical study of 26 patients with trigeminal neuralgia and found the cisternal length of the trigeminal nerve to be $6.33 \pm 2.19 \mathrm{~mm}$ on the affected side and $7.47 \pm 2.72 \mathrm{~mm}$ on the nonaffected side. ${ }^{13}$ Other anatomical variations (e.g., such as atrophy of the trigeminal nerves, a small cerebellopontine angle cistern, and short trigeminal nerves, which are considered typical characteristics of patients with trigeminal neuralgia) were identified by using MRI. ${ }^{13,15}$ Because anatomical variation in patients with trigeminal neuralgia is salient, selection of the target isocenter for radiosurgical treatment of trigeminal neuralgia should be considered.

\section{Effects of Anatomical Variation on Clinical Outcomes}

Our study reports the effects of trigeminal nerve

TABLE 4: Cox regression and logistic regression analysis results

\begin{tabular}{|c|c|c|c|c|c|c|c|}
\hline \multirow[b]{2}{*}{ Analysis } & \multirow[b]{2}{*}{ No. } & \multicolumn{2}{|c|}{ Nerve Length in $\mathrm{mm}$ (cutoff $11 \mathrm{~mm}$ ) } & \multicolumn{2}{|c|}{ Target Length in $\mathrm{mm}$ (cutoff $4 \mathrm{~mm}$ ) } & \multicolumn{2}{|c|}{ Target Ratio in \% (cutoff $36 \%$ ) } \\
\hline & & $p$ Value & $\mathrm{HR}$ (range) & $p$ Value & $\mathrm{HR}$ (range) & $p$ Value & HR (range) \\
\hline \multicolumn{8}{|l|}{ Cox regression } \\
\hline yes & 87 & 0.589 & $1.131(0.723-1.768)$ & 0.604 & $0.893(0.582-1.369)$ & 0.488 & $1.163(0.758-1.783)$ \\
\hline no & 19 & & & & & & \\
\hline no & 73 & & & & & & \\
\hline logistic regression & & & OR (range) & & OR (range) & & OR (range) \\
\hline \multicolumn{8}{|c|}{ facial hypesthesia (BNI II \& III) } \\
\hline yes & 58 & $0.017^{*}$ & $1.245(1.040-1.490)$ & 0.887 & $0.978(0.723-1.325)$ & $0.043^{*}$ & $0.966(0.935-0.999)$ \\
\hline no & 48 & & & & & & \\
\hline
\end{tabular}

${ }^{*} p<0.05$. 


\section{Radiosurgery targeting and anatomical trigeminal nerve variation}

variation on target selection. For trigeminal neuralgia patients at a single treatment center, we administered a constant radiation dose (median $90 \mathrm{~Gy}$; mean $88 \mathrm{~Gy}$ ) at a constant distance from the brainstem $(3.9 \pm 1.3 \mathrm{~mm}$; for $70 \%$ of the patients, GKS was targeted at a location 3-5 $\mathrm{mm}$ from the brainstem). Among several critical variables in GKS used to treat trigeminal neuralgia (including radiation dosage, selective beam channel blocking, and dosage to the brainstem), the target site for radiation has been considered a potential prognostic factor; however, the ideal target remains undetermined. Methodologically, we applied continuous variables in this study to determine the ideal target site. According to our results, anatomical variation of the nerve did not affect the rate and duration of pain relief in either the complete pain relief group or the adequate pain relief group. However, facial hypesthesia occurred more frequently among patients in whom the nerve was longer $(>11 \mathrm{~mm}, \mathrm{p}=0.017)$ and the targeting ratio was lower $(<38 \%, \mathrm{p}=0.043)$; the targeting lengths for the 2 groups were similar $(>4 \mathrm{~mm}, \mathrm{p}=0.887)$.

On the basis of these findings, when treating trigeminal neuralgia by using GKS, anatomical variations, such as variations in nerve length, seem to be more crucial than the absolute distance of radiation. According to our review of relevant research, ours is the first study to investigate the effects of anatomical variation on treatment outcomes by measuring the targeting ratio, which was used to normalize individual variations in nerve length.

\section{Effects of Targeting Different Nerve Zones}

Several recent studies of GKS treatment outcomes have attempted to identify the most effective target zone and improve treatment effectiveness. Several authors have proposed that using a distal target (e.g., the RGZ) can improve pain control, whereas other authors have proposed that using a proximal target (e.g., the DREZ) is more effective. In 2004, Massager et al. recommended using RGZ irradiation and targeting the nerve $5-8 \mathrm{~mm}$ from the brainstem to optimize pain control and minimize complications. ${ }^{10}$ The target distances from the brainstem were measured and classified according to 3 targets: $9.5 \mathrm{~mm}$, $10.2 \mathrm{~mm}$, and $11.3 \mathrm{~mm}$ from the brainstem. These authors concluded that prognostic factors of favorable pain control are use of a target closer to the brainstem, use of a higher radiation dose to the brainstem, and development of facial sensory disturbances. In 2006, Régis et al. further evaluated targeting and reported success rates of $72.7 \%, 84.0 \%$, and $96.6 \%$ when the distance between the isocenter and the emergence of the nerve at the brainstem was longer than $9 \mathrm{~mm}, 7-9 \mathrm{~mm}$, and shorter than $7 \mathrm{~mm}$, respectively. The authors also observed that with RGZ targeting, the closer the isocenter is to the brainstem, the higher is the risk for facial hypesthesia. ${ }^{18}$

Two studies have compared the clinical results obtained by targeting the RGZ with those obtained by targeting the DREZ. Park et al. reported that RGZ targeting yielded greater treatment success and fewer complications than did DREZ targeting..$^{14}$ The authors also observed that frequencies of facial hypesthesia and dry eye syndrome were lower among patients in whom the RGZ was targeted ( $0 \%$ for each) than among patients in whom the DREZ was targeted $(13.1 \%$ and $8.7 \%$, respectively) ( $\mathrm{p}=0.225$ and 0.503 for facial hypesthesia and dry eye syndrome, respectively). However, Matsuda et al. reported considerably different results, which indicated that proximal targeting enabled greater pain control and yielded a lower complication rate. Thus, Matsuda et al. recommended proximal targeting and use of an 80-Gy dose when using GKS to treat trigeminal neuralgia. ${ }^{11}$

$\mathrm{Xu}$ et al. used a proximal target $(50 \%$ isodose line 1-3 $\mathrm{mm}$ anterior to the brainstem vs $30 \%$ isodose line 3-5 $\mathrm{mm}$ anterior to the brainstem) and a maximal dose of 80 Gy and achieved promising results. ${ }^{23}$ Compared with the distal target, the proximal target was associated with a similar rate of pain relief, a longer duration of pain relief, and an increased risk for mild to moderate facial hypesthesia. Facial hypesthesia with BNI scores of II to III was reported for $19(53 \%)$ patients in the proximal target group and $16(25 \%)$ patients in the distal target group $(\mathrm{p}$ $=0.015$ ). Although the ideal target location remains controversial, a greater percentage of patients experienced facial hypesthesia after GKS when the radiation target was closer to the brainstem.

\section{Facial Hypesthesia After 2-Isocenter Radiosurgery}

Flickinger et al. compared the treatment results of trigeminal neuralgia patients who had been randomly selected to undergo retrogasserian GKS by use of either 1 or 2 isocenters. ${ }^{5}$ Their study indicated that increased radiation volume did not improve rates of pain relief; however, the incidence of facial hypesthesia increased. Although the design and purpose of their study differed from those of our study, distinct factors that might increase the incidence of post-GKS facial hypesthesia were observed in both studies. On the basis of our findings, we assumed that the 2-isocenter radiosurgical plan might irradiate more of the proximal part of the trigeminal nerve than does the 1-isocenter plan, which might explain the increased incidence of facial hypesthesia among patients in the 2-isocenter group.

\section{Adjustment of Targeting}

For optimization of the radiation target, anatomical variations should be considered when selecting targets. According to our protocol, the optimal high-dose DREZ irradiation target is located $4 \mathrm{~mm}$ anterior to the brainstem on the trigeminal nerve in patients with a nerve of average length. In patients with a short nerve, use of a radiosurgical target $4 \mathrm{~mm}$ anterior to the brainstem irradiates a more distal segment of the trigeminal nerve. Thus, the clinical effect is similar to irradiating the RGZ. In patients with a long trigeminal nerve, using a target 4 $\mathrm{mm}$ anterior to the brainstem irradiates a more proximal segment of the nerve. Thus, the clinical effects are greater facial hypesthesia and similar rates of pain relief, as reported by Xu et al. ${ }^{23}$ Nevertheless, in our study, increased duration of pain relief among patients who underwent 1-3-mm targeting as well as an increased rate of pain relief and shorter time to response among patients who underwent RGZ targeting were not observed.

Like the results published by Park et al. and $\mathrm{Xu}$ et 
al., ${ }^{14,23}$ our results indicate that the clinical outcomes of trigeminal neuralgia treatment vary according to the segment of the trigeminal nerve that is irradiated. When 90 Gy of radiation was administered to the DREZ, the risk for trigeminal neuropathy was substantially higher among patients who had longer nerves and lower targeting ratios. Although most facial hypesthesia reported did not irritate the patient and the hypesthesia was considered to be a predictor of successful neuropathy treatment, ${ }^{8,10,18}$ the risk for facial hypesthesia significantly increased by $24.5 \%$ with each $1-\mathrm{mm}$ increase in nerve length and significantly increased by $3.4 \%$ with each $1 \%$ decrease in targeting ratio (with adjustment for sex and age, Table 4), whereas the pain relief rates remained constant.

Our results demonstrate that adjusting the target based on the targeting ratio and keeping the target slightly distal to a targeting ratio greater than $36 \%$ might reduce the rate of facial hypesthesia and enable rates of pain control similar to those obtained by using DREZ targeting (Fig. 2). However, for Taiwanese patients, the cutoff value for reducing facial hypesthesia (nerve length $<11 \mathrm{~mm}$ and targeting ratio $>36 \%$ ) is limited to DREZ targeting. Future studies should evaluate the GKS effects of anatomical variation among patients of different races.

\section{Study Limitations}

Our study was retrospective, and patient selection and treatment biases that reflect the policies and practices of the clinicians and our medical center may have been inherent to the design. All patients were Asian. The validity of our findings for non-Asian populations might require further investigation.

To calculate the length of the trigeminal nerve, we simplified the length as 1 straight line between 2 points measured according to coordinates on a $1.5-\mathrm{mm}$ thinsection MR image. However, the structure of nerves is 3 dimensional. Errors might have been introduced during the identification of the 2 coordinates: the midpoint of the junction between the trigeminal nerve and the brainstem and the midpoint of the trigeminal nerve as it reaches the dural edge.

For verification of the differences in the extent and duration of pain relief and the complication rates for each GKS target, a randomized trial comparing the target locations and adequately controlling for other variables (e.g., etiology of facial pain, prior procedures) should be conducted. However, randomized trials of radiosurgery performed to treat trigeminal neuralgia are costly, logistically challenging, and rarely approved.

\section{Conclusions}

This study provided clinical outcome data with regard to GKS performed to treat trigeminal neuralgia by using a maximal dose of $90 \mathrm{~Gy}$. Complete pain relief and adequate pain relief were achieved for $59(56 \%)$ and $87(82 \%)$ patients, respectively. After GKS, facial hypesthesia with a BNI score of II or III developed in 57 (54\%) patients (BNI Score II for 44 [42\%] and BNI Score III for 13 [12\%]). All patients underwent procedures targeting the DREZ (median targeting length $3.8 \mathrm{~mm}$ anterior to the brainstem). Although the patients in this study exhibited individual anatomical variations, the rates of pain relief did not differ. We observed more facial hypesthesia among patients with a longer nerve $(>11 \mathrm{~mm})$ or those for whom the targeting ratio was lower $(<36 \%)$. Adjusting the GKS target according to the targeting ratio, especially for patients with long nerves, might lower rates of facial hypesthesia and enable maintenance of effective pain control.

\section{Acknowledgments}

We are grateful to Ms. Ya-Fang Hsu, Ms. Feng-Chiao Lee, and Ms. Hush-Chen Huang for their assistance with data collection.
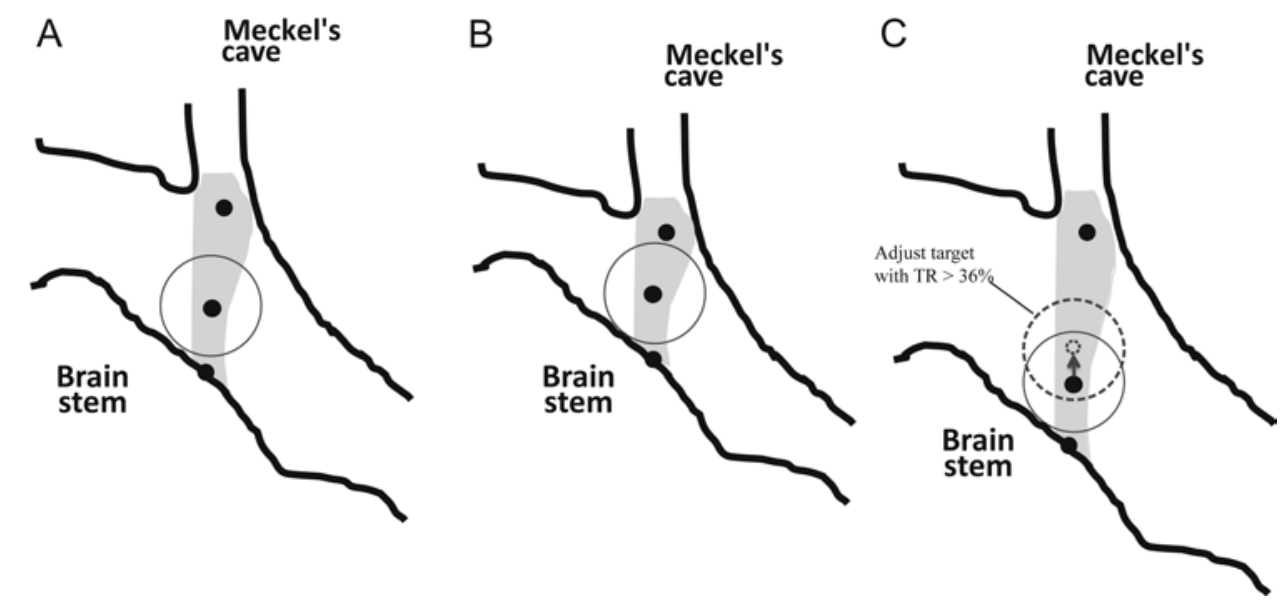

FIG. 2. Effect of anatomical variation on fixed-distance targeting plans. In our protocol, for a patient with a trigeminal nerve of average length, the radiosurgical target is placed $4 \mathrm{~mm}$ anterior to the brainstem with a $20 \%$ isodose line to brainstem (black dot with solid circle) (A). For patients with short nerves, using a target $4 \mathrm{~mm}$ anterior to the brainstem (black dot with solid circle) causes irradiation of a more distal segment of the trigeminal nerve (B). In patients with long nerves, using a target $4 \mathrm{~mm}$ anterior to the brainstem (black dot with solid circle) causes irradiation of a more proximal segment of the trigeminal nerve (C). Adjusting the GKS target according to a targeting ratio (TR) greater than 36\% (a modified target moved anteriorly [arrow and small dashed circle] with a $20 \%$ isodose line [large dashed circle]), especially in patients with a long nerve, might lower rates of facial hypesthesia and enable maintenance of effective pain control. 


\section{Radiosurgery targeting and anatomical trigeminal nerve variation}

\section{Disclosure}

The authors report no conflict of interest concerning the materials or methods used in this study or the findings specified in this paper.

Conception and design: Hung. Acquisition of data: Hung, Lee, Liu. Analysis and interpretation of data: Hung, Lee, Liu, Chung. Drafting the article: Yang, Hung. Critically revising the article: Yang, Hung, Chung. Reviewed submitted version of manuscript: Yang. Approved the final version of the manuscript on behalf of all authors: Yang. Statistical analysis: Lee. Administrative/technical/ material support: Liu, Chung, Pan. Study supervision: Pan.

\section{References}

1. Alexander E III, Lindquist C: Special indications: radiosurgery for functional neurosurgery and epilepsy, in Alexander E III, Loeffler JS, Lunsford LD (eds): Stereotactic Radiosurgery. New York: McGraw-Hill, 1993, pp 221-225

2. Alpert TE, Chung CT, Mitchell LT, Hodge CJ, Montgomery CT, Bogart JA, et al: Gamma knife surgery for trigeminal neuralgia: improved initial response with two isocenters and increasing dose. J Neurosurg 102 Suppl:185-188, 2005

3. Balamucki CJ, Stieber VW, Ellis TL, Tatter SB, Deguzman AF, McMullen KP, et al: Does dose rate affect efficacy? The outcomes of 256 gamma knife surgery procedures for trigeminal neuralgia and other types of facial pain as they relate to the half-life of cobalt. J Neurosurg 105:730-735, 2006

4. Dhople AA, Adams JR, Maggio WW, Naqvi SA, Regine WF, Kwok Y: Long-term outcomes of Gamma Knife radiosurgery for classic trigeminal neuralgia: implications of treatment and critical review of the literature. Clinical article. J Neurosurg 111:351-358, 2009

5. Flickinger JC, Pollock BE, Kondziolka D, Phuong LK, Foote RL, Stafford SL, et al: Does increased nerve length within the treatment volume improve trigeminal neuralgia radiosurgery? A prospective double-blind, randomized study. Int J Radiat Oncol Biol Phys 51:449-454, 2001

6. Kakizawa Y, Seguchi T, Kodama K, Ogiwara T, Sasaki T, Goto T, et al: Anatomical study of the trigeminal and facial cranial nerves with the aid of 3.0-tesla magnetic resonance imaging. J Neurosurg 108:483-490, 2008

7. Kondziolka D, Lacomis D, Niranjan A, Mori Y, Maesawa S, Fellows W, et al: Histological effects of trigeminal nerve radiosurgery in a primate model: implications for trigeminal neuralgia radiosurgery. Neurosurgery 46:971-977, 2000

8. Kondziolka D, Zorro O, Lobato-Polo J, Kano H, Flannery TJ, Flickinger JC, et al: Gamma Knife stereotactic radiosurgery for idiopathic trigeminal neuralgia. Clinical article. J Neurosurg 112:758-765, 2010

9. Leksell L: The stereotaxic method and radiosurgery of the brain. Acta Chir Scand 102:316-319, 1951

10. Massager N, Lorenzoni J, Devriendt D, Desmedt F, Brotchi J, Levivier M: Gamma knife surgery for idiopathic trigeminal neuralgia performed using a far-anterior cisternal target and a high dose of radiation. J Neurosurg 100:597-605, 2004

11. Matsuda S, Serizawa T, Nagano O, Ono J: Comparison of the results of 2 targeting methods in Gamma Knife surgery for trigeminal neuralgia. J Neurosurg 109 Suppl:185-189, 2008

12. Nicol B, Regine WF, Courtney C, Meigooni A, Sanders M,
Young B: Gamma knife radiosurgery using $90 \mathrm{~Gy}$ for trigeminal neuralgia. J Neurosurg 93 Suppl 3:152-154, 2000

13. Parise M, Acioly MA, Ribeiro CT, Vincent M, Gasparetto EL: The role of the cerebellopontine angle cistern area and trigeminal nerve length in the pathogenesis of trigeminal neuralgia: a prospective case-control study. Acta Neurochir (Wien) 155:863-868, 2013

14. Park SH, Hwang SK, Kang DH, Park J, Hwang JH, Sung JK: The retrogasserian zone versus dorsal root entry zone: comparison of two targeting techniques of gamma knife radiosurgery for trigeminal neuralgia. Acta Neurochir (Wien) 152: $1165-1170,2010$

15. Park SH, Hwang SK, Lee SH, Park J, Hwang JH, Hamm IS: Nerve atrophy and a small cerebellopontine angle cistern in patients with trigeminal neuralgia. Clinical article. J Neurosurg 110:633-637, 2009

16. Pollock BE, Phuong LK, Foote RL, Stafford SL, Gorman DA: High-dose trigeminal neuralgia radiosurgery associated with increased risk of trigeminal nerve dysfunction. Neurosurgery 49:58-64, 2001

17. Pollock BE, Phuong LK, Gorman DA, Foote RL, Stafford SL: Stereotactic radiosurgery for idiopathic trigeminal neuralgia. J Neurosurg 97:347-353, 2002

18. Régis J, Metellus P, Hayashi M, Roussel P, Donnet A, BilleTurc F: Prospective controlled trial of gamma knife surgery for essential trigeminal neuralgia. J Neurosurg 104:913-924, 2006

19. Régis J, Tuleasca C: Editorial. Fifteen years of Gamma Knife surgery for trigeminal neuralgia in the Journal of Neurosurgery: history of a revolution in functional neurosurgery. $\mathbf{J}$ Neurosurg 115 Suppl:2-7, 2011

20. Rogers CL, Shetter AG, Fiedler JA, Smith KA, Han PP, Speiser BL: Gamma knife radiosurgery for trigeminal neuralgia: the initial experience of The Barrow Neurological Institute. Int J Radiat Oncol Biol Phys 47:1013-1019, 2000

21. Sheehan J, Pan HC, Stroila M, Steiner L: Gamma knife surgery for trigeminal neuralgia: outcomes and prognostic factors. J Neurosurg 102:434-441, 2005

22. Urgosik D, Liscak R, Novotny J Jr, Vymazal J, Vladyka V: Treatment of essential trigeminal neuralgia with gamma knife surgery. J Neurosurg 102 Suppl:29-33, 2005

23. Xu Z, Schlesinger D, Moldovan K, Przybylowski C, Sun X, Lee CC, et al: Impact of target location on the response of trigeminal neuralgia to stereotactic radiosurgery. Clinical article. J Neurosurg 120:716-724, 2014

24. Young B, Shivazad A, Kryscio RJ, St Clair W, Bush HM: Long-term outcome of high-dose Gamma Knife surgery in treatment of trigeminal neuralgia. Clinical article. J Neurosurg 119:1166-1175, 2013

Manuscript submitted June 30, 2014.

Accepted July 29, 2014.

Please include this information when citing this paper: DOI: 10.3171/2014.7.GKS141432.

Address correspondence to: Huai-Che Yang, M.D., Department of Neurosurgery, Neurological Institute, Taipei Veterans General Hospital, 17 F., No. 210, Sec. 2, Shi-Pai Rd., Peitou, Taipei 11217 , Taiwan.email: wade012@gmail.com. 